HARDY-RAMANUJAN JOURNAL 36 (2013), 1-7

\title{
ON THE CLASS NUMBER FORMULA OF CERTAIN REAL QUADRATIC FIELDS
}

\author{
K. CHAKRABORTY, S. KANEMITSU AND T. KUZUMAKI
}

To Professor Tsuyoshi Uehara, with great respect and friendship

ABSTRACT. In this note we give an alternate expression of class number formula for real quadratic fields with discriminant $d \equiv 5 \bmod 8$.

\section{INTRODUCTION}

Let $p$ be an odd prime with $p \equiv 1 \bmod (4)$ and $\varepsilon$ be the fundamental unit of $\mathbb{Q}(\sqrt{p})$. It is well known that the class number $h$ in this case is given by

$$
h=\frac{\sqrt{p}}{2 \log \varepsilon} L(p)
$$

where $L(p)=\sum_{k=1}^{\infty}\left(\frac{p}{k}\right) k^{-1}$. From here one can deduce classical Dirichlet's class number formula

$$
\varepsilon^{2 h}=\frac{\prod_{n}\left(1-\theta^{n}\right)}{\prod_{v}\left(1-\theta^{v}\right)}
$$

where $\theta$ is the primitive $p$-th root of unity; $v$ and $n$ run over the quadratic residues and non-residues of $p$ respectively in the interval $(0, p)$. This formula exhibit class number $h$ of the corresponding real quadratic field in a "transcendental" manner. A somewhat simpler formula was proved by P. Chowla [3] in the case of prime discriminant of the form $p \equiv 5 \bmod 8$. She took the square root of the above classical formula to derive her formula.

We derive an alternate class number formula for real quadratic fields with discriminant $d \equiv 5 \bmod 8$. We appeal to another form of the class number formula for $\varepsilon^{h}$ and transform it using another result of Dirichlet for 1/4-th character sum, in such a way that the obtained result has the effect of taking the square root of the class number formula for $\varepsilon^{2 h}$ as was done by P. Chowla. Our result can elucidate other generalisations of Mitsuhiro, Nakahara and Uehara for $\varepsilon^{2 h}$ for general real quadratic fields.

2010 Mathematics Subject Classification. Primary: 11R29, Secondary: 11R60.

Key words and phrases. Real quadratic field, class number formula. 


\section{SquARe Root of the Class NUMBer FORMula}

Let $d$ be a positive discriminant. We denote by $h$ the class number of the real quadratic field $\mathbb{Q}(\sqrt{d})$ and by $\varepsilon>1$ its fundamental unit. We consider the following version([2, Theorem3, p. 246]) of the classical class number formula of Dirichlet

$$
\varepsilon^{h}=\frac{\prod_{0<n<d / 2} \sin \frac{\pi n}{d}}{\prod_{0<v<d / 2} \sin \frac{\pi v}{d}},
$$

where $v$ and $n$ will always denote integers such that the Kronecker symbols $\left(\frac{v}{d}\right)=1$ and $\left(\frac{n}{d}\right)=-1$ respectively.

In 1966, P. Chowla [3] showed that for $d=p \equiv 5 \bmod 8$, one has:

$$
\varepsilon^{h}=(-1)^{m} \prod_{0<n<p / 2}\left[2 \cos \frac{2 \pi}{p}\left\{\frac{n}{2}\right\}\right]
$$

where $m$ denotes the number of odd $n$ 's with $n \leq p / 2$ and for any odd positive integer $d$, the symbol $\left\{\frac{n}{2}\right\}$ denotes the solution $k$ of congruence equation $2 X \equiv n \bmod d$ with $0<k<d$. In the same paper she also shows that $m$ can be computed via the identity:

$$
m=\frac{1}{2}\left[\frac{p-1}{4}+H\right],
$$

where $H$ denotes the class number of the imaginary quadratic field $\mathbb{Q}(\sqrt{-d})$.

The goal of this note is to extend Chowla's formula to the case when $d$ is not necessarily prime. More precisely, we will prove the following:

Theorem 2.1. Let $d \equiv 5 \bmod 8$ be a positive discriminant and let $h$ and $\varepsilon>1$ be the class number and the fundamental unit of $\mathbb{Q}(\sqrt{d})$, respectively. Furthermore let $H$ denote the class number of $\mathbb{Q}(\sqrt{-d})$. Then

$$
\begin{gathered}
\varepsilon^{h}=(-1)^{m} \prod_{v<\frac{d}{2}} 2 \cos \frac{2 \pi}{d}\left\{\frac{v}{2}\right\}, \quad \varepsilon^{-h}=(-1)^{l} \prod_{n<\frac{d}{2}} 2 \cos \frac{2 \pi}{d}\left\{\frac{n}{2}\right\} \\
m=\frac{1}{2}\left(\frac{\varphi(d)}{2}-\varphi\left(d, \frac{d}{4}\right)+\frac{1}{2} H\right), l=\frac{1}{2}\left(\frac{\varphi(d)}{2}-\varphi\left(d, \frac{d}{4}\right)-\frac{1}{2} H\right)
\end{gathered}
$$

where $\varphi(a, b)$ denote the number of rational integers $k$ such that $0<k<b$ and $(k, a)=1$.

Let $T, U, T_{1}$ and $U_{1}$ be the integers defined by the identities

$$
\frac{T+U \sqrt{d}}{2}=\varepsilon^{2 h}, \quad \frac{T_{1}+U_{1} \sqrt{d}}{2}=\varepsilon^{h} .
$$


Then,

$$
\begin{aligned}
T_{1} & =\varepsilon^{h}+N(\varepsilon)^{h} \varepsilon^{-h} \\
& =(-1)^{m}\left(\prod_{v<\frac{d}{2}}\left(\theta^{\left\{\frac{v}{2}\right\}}+\theta^{-\left\{\frac{v}{2}\right\}}\right)+\prod_{n<\frac{d}{2}}\left(\theta^{\left\{\frac{n}{2}\right\}}+\theta^{-\left\{\frac{n}{2}\right\}}\right)\right)
\end{aligned}
$$

Since $d \equiv 1 \bmod 4$, the function $\varphi\left(d, \frac{d}{4}\right)$ can be computed as in [6]:

$$
\varphi\left(d, \frac{d}{4}\right)= \begin{cases}\frac{\varphi(d)}{4}+2^{r-2} & \text { if any prime } p \mid d \text { are } p \equiv 3 \quad \bmod 4 \\ \frac{\varphi(d)}{4} & \text { otherwise, }\end{cases}
$$

where $r$ is the number of prime factors of $d$.

In a later paper Chowlas' [4], still in the case $p \equiv 5 \bmod 8$ and with added assumption that $h=1$, proved that $T$ is the least positive residue $\left(\bmod 2^{p}-1\right)$ of

$$
(-1)^{m}\left[\prod_{v<\frac{p}{2}}\left(2^{v}+2^{-v}\right)+\prod_{v<\frac{p}{2}}\left(2^{2 v}+2^{-2 v}\right)\right] .
$$

Another classical formulation of the Dirichlet class number formula states that,

$$
\varepsilon^{2 h}=\frac{F_{-}(\theta)}{F_{+}(\theta)},
$$

where $\theta=e^{\frac{2 \pi i}{d}}$ denotes the $d$-th root of 1 with lowest argument and

$$
F_{ \pm}(X)=\prod_{\substack{a<d \\\left(\frac{a}{d}\right)= \pm 1}}\left(X^{a}-1\right) .
$$

In [6], Mitsuhiro, Nakahara and Uehara proved a result which allows to compute $T$ for a general discriminant $d \neq 12$. If $\Phi_{d}(x)$ denotes the $d$-cyclotomic polynomial, then their result states that $T$ is the least positive residue $\left(\bmod \Phi_{d}(2)\right)$ of the quantity:

$$
\frac{F_{-}(2)}{F_{+}(2)}+\frac{F_{+}(2)}{F_{-}(2)}
$$

A consequence of Theorem 2.1 is:

Corollary 2.2. Suppose $d \equiv 5 \bmod 8$. Then $T_{1}$ is the least positive residue mod $\Phi_{d}(2)$ of

$$
(-1)^{m}\left(\prod_{v<\frac{d}{2}}\left(2^{v}+2^{-v}\right)+\prod_{v<\frac{d}{2}}\left(2^{2 v}+2^{-2 v}\right)\right)
$$


Remark 1. These results may have relation with the class number relation for the biquadratic field ([5]) $\mathbb{Q}(i, \sqrt{d})$ for a square-free integer $d \equiv 1 \bmod 4$, which is a Galois extension of $\mathbb{Q}$ and its Galois group is $V_{4}$ with subfields $\mathbb{Q}(\sqrt{d}), \mathbb{Q}_{4}=\mathbb{Q}(\sqrt{-4})$ and $\mathbb{Q}(\sqrt{-d})$.

\section{Proofs}

Proof of Theorem 2.1. The Kronecker symbol is even when $d>0$. Hence (1) follows from (6) by rewriting it as

$$
\varepsilon^{2 h}=\frac{\prod_{0<n<d} \sin \frac{\pi n}{d}}{\prod_{0<v<d} \sin \frac{\pi v}{d}}=\frac{\prod_{0<n<d / 2} \sin \frac{\pi n}{d} \sin \frac{\pi(d-n)}{d}}{\prod_{0<v<d / 2} \sin \frac{\pi v}{d} \sin \frac{\pi(d-v)}{d}}=\left(\frac{\prod_{0<n<d / 2} \sin \frac{\pi n}{d}}{\prod_{0<v<d / 2} \sin \frac{\pi v}{d}}\right)^{2} .
$$

As $\left(\frac{2}{d}\right)=-1$, the non-residues are given in the form $2 v([3, \mathrm{p} .54])$. Hence (1) may be written as

$$
\varepsilon^{h}=\prod \frac{\sin \frac{2 \pi v}{d}}{\sin \frac{\pi v}{d}}=\prod_{0<v<d / 2} 2 \cos \frac{\pi v}{d}
$$

Noting that $\left\{\frac{v}{2}\right\}$ also means the least positive residue $\bmod d$ of $2^{-1} v$, where $2^{-1}$ is the inverse of $2 \bmod d$ :

$$
\left\{\frac{v}{2}\right\}=2^{-1} v-d\left[\frac{2^{-1} v}{d}\right]
$$

$[x]$ denotes the integral part of $x$. Thus we can express $\left\{\frac{v}{2}\right\}$ more concretely as

$$
\left\{\frac{v}{2}\right\}=\left\{\begin{array}{lll}
\frac{v}{2} & v & \text { even } \\
\frac{v+d}{2} & v & \text { odd }
\end{array}\right.
$$

Hence, if we replace $v$ in (8) by $\left\{\frac{v}{2}\right\}$, then those terms with odd $v$ are negative and there are $m$ of them, where $m$ indicates the number of odd residues in $\left(0, \frac{d}{2}\right)$. Hence it follows that

$$
\varepsilon^{h}=(-1)^{m} \prod_{0<v<d / 2} 2 \cos 2 \pi\left\{\frac{v}{2}\right\}
$$

The remaining part is the same as in [3] and we prove as she has done by elementary argument that,

$$
m=\frac{1}{2}\left\{\frac{\varphi(d)}{2}-\varphi\left(d, \frac{d}{4}\right)+\frac{1}{4} S_{1 / 4}\right\} .
$$

Where $2 S_{1 / 4}=H$, which is a form of Dirichlet class number formula for $\mathbb{Q}(\sqrt{-d})$. Moving in a similar fashion we can prove the case of $\varepsilon^{-h}$ and $l$.

We now have the proof modulo the fact that $N(\varepsilon)^{h}=1$ or -1 and we prove it in the course of the proof of Corollary 2.2. 
Proof. (Proof of Corollary 2.2) Our proof is similar to Chowlas' [4, Proof of Theorem 1] by appealing to the Gauss sum

$$
\sqrt{d}=\sum_{a<d} \chi_{d}(a) \theta^{a}=\sum_{v<d} \theta^{v}-\sum_{n<d} \theta^{n}
$$

This follows by taking the square root of the formula

$$
(-1)^{\frac{d-1}{2}} d=\left(\sum_{a<d} \chi_{d}(a) \theta^{a}\right)^{2}
$$

Substituting (11) in (2), we deduce that,

$$
\frac{1}{2}\left(T_{1}+U_{1}\left(\sum_{v<d} \theta^{v}-\sum_{n<d} \theta^{n}\right)\right)=\varepsilon^{h}=(-1)^{m} \prod_{v<\frac{d}{2}}\left(\theta^{\left\{\frac{v}{2}\right\}}+\theta^{-\left\{\frac{v}{2}\right\}}\right) .
$$

Changing $\theta$ in (11) by $\theta^{4}$ and $\theta^{2}$, respectively, thereby noting that $2\left\{\frac{v}{2}\right\} \equiv v \bmod d$ and $\chi_{d}(2)=-1$, we obtain two expressions for $d$. Substituting this in (12), we deduce that

$$
\varepsilon^{h}=(-1)^{m} \prod_{v<\frac{d}{2}}\left(\theta^{2 v}+\theta^{-2 v}\right)
$$

and

$$
\varepsilon^{\prime h}=(-1)^{m} \prod_{v<\frac{d}{2}}\left(\theta^{v}+\theta^{-v}\right)
$$

where $\varepsilon^{\prime}$ is the conjugate of $\varepsilon$. Now summing (13) and (14) we have,

$$
T_{1}=\varepsilon^{h}+\varepsilon^{\prime h},
$$

which is (3).

The number of $v$ such that $\theta^{v}+\theta^{-v}<0$ and $0<v<d / 2$ is $l$, and $\varepsilon^{\prime}=N(\varepsilon) \varepsilon^{-1}$. Thus $N(\epsilon)^{h}=(-1)^{m+l}$, and this completes the proof of Theorem 2.1.

It remains to prove that $T_{1}$ is the least positive residue $\bmod \Phi_{d}(2)$. The right-hand side is estimated by

$$
T_{1}<2^{\frac{\varphi(d)}{2}}+1<2^{\varphi(d)-2}
$$

thus it is less than $\Phi_{d}(2)$ (cf. [6, p.101]). Finally, the polynomial

$$
P(X)=X^{d^{2}}\left(T_{1}-(-1)^{m}\left(\prod_{v<\frac{d}{2}}\left(X^{v}+X^{-v}\right)+\prod_{v<\frac{d}{2}}\left(X^{2 v}+X^{-2 v}\right)\right)\right)
$$

is divisible by $\Phi_{d}(X)$ because it vanishes at $X=\theta$ and $\Phi_{d}(X)$ is the minimal polynomial for $\theta$. 
In [6], Mitsuhiro, Nakahara and Uehara considered a generalization of another result of Chowlas for $\varepsilon^{2 h}$. Let $T=T_{2}, U=U_{2}$ be defined by

$$
\frac{T+U \sqrt{d}}{2}=\varepsilon^{2 h}
$$

Then their theorem states that,

$$
T=T_{2}=\varepsilon^{2 h}+\varepsilon^{-2 h}
$$

is the least positive residue $\bmod \Phi_{d}(2)$ of $\frac{F_{-}(2)}{F_{+}(2)}+\frac{F_{+}(2)}{F_{-}(2)}$. Their proof is similar to that of Chowlas' except for one point in which they need to prove a key lemma which shows that $\left|\theta^{ \pm n}\right|<\sqrt{2}$ for non-residue $n$ in the first $1 / 4$-th interval. Thus establishing that the above value is less than $\Phi_{d}(2)$.

We conclude by showing that with Dirichlet's formula for the $1 / 4$-th sum one can give a simpler proof of their Lemma [6, Lemma].

Proposition 3.1. For any discriminant d except for 5, 8, 12, 24, there exists a nonresidue mod $d$ in the first $1 / 4$-th interval.

Proof. Assume that $\chi_{d}(a)=1$ for all $a$ in $0<<\frac{1}{4} d$. Then $\chi_{d}(a)=-1$ in $\frac{1}{4} d<<\frac{3}{4} d$ and $\chi_{d}(a)=1$ in $\frac{3}{4} d<<d$. We distinguish cases following [6].

- $d \equiv 1 \bmod 4$ with $d>5 . \chi_{d}(2)=1$ and $0<2<\frac{d}{4}$. Hence there is an $\alpha$ such that $\frac{d}{2}<2^{\alpha}<\frac{d}{4}$ for which $\chi_{d}\left(2^{\alpha}\right)=1$, a contradiction.

- $d=4 f, f \equiv 3 \bmod 4, f>3 . f^{2} \equiv 1 \bmod 4$ and $\chi_{d}(f+2)=-1$, so that $(f+2)^{3} \equiv 3 f+8 \bmod d$. For $f=7,3 f+8 \equiv 1 \bmod d$ while for $f>7$, $\frac{3}{4} d<3 f+8<d$ and $\chi_{d}(3 f+8)=-1$, a contradiction.

- $d=8 f$. There are two cases. If $f \equiv 1 \bmod 4$, then $\chi_{d}(3 f-2)=-1$ and $(3 f-2)^{3} \equiv f-8 \bmod d$, so that $\chi_{d}(f-8)=-1$. If $f \equiv 3 \bmod 4$, then $3 f+2$ works.

Remark 2. Noting that $T_{1}^{2}=T-2$ (which follows from the Pell equation $T_{1}^{2}-U_{1}^{2} d=$ 4), we see that to treat the general case of Mitsuhiro, Nakahara and Uehara, we can work with (5) as in Chowlas' and appeal to their Lemma.

Acknowledgements. The authors would like to express their sincere gratitude to the anonymous referee for carefully going through the earlier version of the manuscript and suggesting meaningful changes. This has helped improving the presentation.

\section{REFERENCES}

[1] B. C. Berndt, Classical theorems on quadratic residues, Ensein. Math. (2) 22 (1976), 261-304.

[2] Z. Borevič and I. Šafarevič, The theory of numbers, Izd. Nauka, Moscow 1964; Number Theory, Academic Press 1966. 
[3] P. Chowla, On the class-number of real quadratic fields, J. Reine Angew. Math. 230 (1968), 51-60.

[4] P. Chowla and S. Chowla, Formulae for the units and class-numbers of real quadratic fields, J. Reine Angew. Math. 230 (1968), 61-65.

[5] H. Cohen, q-identities for Maass waveforms, Invent. Math. 91 (1988), 409-422.

[6] T. Mitsuhiro, T. Nakahara and T. Uehara, The class number formula of a real quadratic field and an estimate of the value of a unit, Canad. Math. Bull. 38 (1995), 98-103.

Kalyan chakraborty, Harish-Chandra Research Institute, Chhatnag Road, Jhunsi, Allahabad 211019, India.

E-mail address: kalyan@hri.res.in

Shigeru Kanemitsu, Graduate School of Advanced Technology, Kinki University, IIZUKA, FUKUOKA 820-8555, JAPAN.

E-mail address: kanemitu@fuk.kindai.ac.jp

Takako Kuzumaki, Faculty of Engineering, Gifu University, Gifu 501-1193, Japan. E-mail address: kuzumaki@gifu-u.ac.jp 